\title{
El espacio de la fiesta y los lugares de la tradición. Tensiones y vínculos en torno a la desaparición de un sistema de cargos urbano y sus mayordomías en el barrio de San Miguelito de la ciudad de San Luis Potosí. Por David Madrigal González. San Luis Potosí: El Colegio de San Luis, 2or6, I65 p.
}

\author{
Víctor Eder Morales López \\ El Colegio Mexiquense \\ vmorales@cmq.edu.mx \\ do \\ DOI: I0.2490I/rehs.v40iI57.444
}

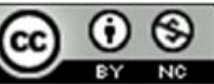

El espacio de la fiesta y los lugares de la tradición. Tensiones y vínculos en torno a la desaparición de un sistema de cargos urbano y sus mayordomías en el barrio de San Miguelito de la ciudad de San Luis Potosí. Por David Madrigal González. San Luis Potosí: El Colegio de San Luis, 2or6, I65 p. por Victor Eder Morales López se distribuye bajo una Licencia Creative Commons Atribución-NoComercial 4.0 Internacional.

La obra aquí reseñada es resultado de la investigación realizada entre 2003 y 2004, y de 201 a a 2013, por David Madrigal, investigador del programa de estudios antropológicos adscrito a El Colegio de San Luis. Las líneas de investigación que trabaja son los conflictos socioambientales, acción colectiva, movilizaciones sociales, tribus urbanas y sistemas de cargos urbanos.

Esta investigación de corte antropológico, es un ejemplo del diálogo interdisciplinario entre ciencias humanísticas y sociales. Para este caso, el autor logra sostener un diálogo donde converge la antropología con la historia, la economía, la geografía y la sociología. Para entender la necesidad de un debate interdisciplinario como el propuesto por Madrigal, se pueden responder preguntas como ¿cuáles son los alcances de la interdisciplinariedad en el trabajo de investigación?, ¿cómo logra conjugar el autor distintas disciplinas en su trabajo de investigación? y ¿el autor puede alcanzar su objetivo mediante un trabajo interdisciplinario?

La obra puede ser leída por dos tipos de público: por un lado, las personas interesadas en las manifestaciones populares. Por otro, los estudiosos que incursionan en el tema de los sistemas de cargos, desde perspectivas antropológicas, históricas o sociológicas. El lenguaje que usa Madrigal es claro, que es fundamental para tener acceso al texto desde diferentes ópticas disciplinarias. 
Desde el primer apartado se puede advertir la noción espacial del barrio de San Miguelito con una perspectiva histórica, la cual remite al periodo virreinal con la fundación del pueblo de San Luis en I576. Este enfoque histórico permite conocer el proceso de conformación de sus barrios, el barrio de San Miguel se fundó en 597 (posteriormente San Miguelito), ubicado al sur de lo que era el pueblo de San Luis.

El texto se complementa con elementos de demografía, economía y política que ayudan a contextualizar el periodo colonial y la etapa decimonónica del barrio de San Miguelito. Esto da pauta para contrastar los periodos históricos de conformación del barrio con la modernidad y los problemas derivados de la ausencia de políticas urbanas.

Los aportes que se pueden manifestar recíprocamente entre diversas disciplinas ayudan a enriquecer el trabajo del investigador. Sin embargo, en el estudio de Madrigal se pueden advertir un par de ausencias. Un ejemplo es el sustento histórico del estudio, el cual tiene mayor atención en trabajos historiográficos. Las obras que componen ese corpus historiográfico son de autores como Primo Feliciano Velázquez, Antonio Cabrera, José Alberto Juárez Miranda, Alfonso Martínez Rosales o Raymundo Reina Alviso.

En ese sentido podría resultar enriquecedor un acercamiento directo a fuentes de archivo, las cuales podrían contener pistas que otros investigadores no hayan notado. Para esto, los archivos locales o incluso documentos que hayan elaborado los miembros del sistema de cargos del barrio de San Miguelito podrían ser fuentes de importantes datos sobre la conformación del barrio, o posiblemente sobre diferentes procesos propios del sistema de cargos.

Como menciona el título, uno de los intereses de Madrigal es la tensión en torno a la desaparición del sistema de cargos en el barrio de San Miguelito. Es evidente la utilidad de la ciencia histórica para conocer el proceso que fue determinando las tensiones que el autor investiga. Por otro lado, los elementos que proporciona la antropología son cruciales para entender la actualidad de la fiesta en el espacio social del barrio de San Miguelito. Para eso el autor enfatiza la participación de los vecinos de cada cuartel del barrio, la intensidad de la participación, algunos obstáculos, los vínculos de los mayordomos con los vecinos para los preparativos y difusión de la fiesta. Es interesante cómo el autor guía a su lector señalando el método utilizado para medir la intensidad de participación de los vecinos de cada cuartel.

Su método consistió en la cuantificación de los adornos que se colocaban en las calles y la complementó observando las actividades de los vecinos. Es en esta parte donde podría haber un segundo vacío. Cabe la posibilidad de que la cantidad de adornos de cada cuartel responda a otros factores independientemente de la intensidad de la participación, como el poder adquisitivo de los vecinos y diferentes alternativas de cooperar, además de que no es un acercamiento directo con los pobladores.

La metodología de cuantificación y de observación se podría reforzar mediante un acercamiento directo con los vecinos. Ello se puede lograr con la aplicación de instrumentos empíricos 
enfocados en medir directamente la participación vecinal. Ese tipo de interacción directa podría proveer de datos más concretos respecto a su participación en la fiesta.

La historia y la geografía aportaron a Madrigal las bases para comprender la formación del espacio social que estudia y posteriormente transportar esas bases al campo de la antropología. Con el uso de su propia metodología logró hacer un contraste interesante con el presente de la fiesta, resaltando uno de los principales problemas que enfrenta: la desaparición del sistema de cargos en el barrio.

El autor explora los elementos que componen la fiesta antes de la desaparición de los sistemas de cargos, describiendo como fueron los últimos recorridos previos y convites con los mayordomos, así como otras actividades a cargo de los mayordomos como la bajada del santo, las últimas serenatas y la fiesta de San Miguel que se celebra el 29 de septiembre.

A lo largo del estudio que ofrece Madrigal, se observa que las tensiones en torno al sistema de cargos surgen desde problemas contemporáneos como la globalización. Problema que se acentúa por la pérdida de espacios simbólicos en el barrio de San Miguelito ante empresas transnacionales que transforman los espacios tradicionales en espacios comerciales.

Se puede suponer que Madrigal propone el análisis de las formas en que la globalización incide en el contexto local de barrio de San Miguelito. Sin embargo, su objetivo consiste en abordar algunas formas de cómo se está viviendo en el contexto local en tiempos globalizados. En ese sentido, el autor logra alcanzar su objetivo al analizar la forma en que viven la fiesta los habitantes del barrio de San Miguelito. Un enfoque interdisciplinario le permite concretar el objetivo al desentrañar las tensiones que surgen en el barrio por los efectos de la globalización. Elementos como identidad, relaciones y vínculos sociales fungen como mecanismos de resistencia ante la desaparición del sistema de cargos barrial.

Al perder terreno ante la globalización, el sistema de cargos que describe Madrigal está en riesgo de desaparecer. No obstante, el autor no descarta la posibilidad de que más adelante surja un sistema reorganizado y reestructurado. Posiblemente esa nueva organización presente un nuevo rostro, pero se espera que cumpla las mismas funciones barriales del sistema de cargos que en la actualidad agoniza. 